\title{
The impact of routine pulse oximetry use on outcomes in COVID-19-infected patients at increased risk of severe disease: A retrospective cohort analysis
}

\author{
N Nematswerani, ${ }^{1}$ MB ChB, MPharmMed, MSc Clinical Epidemiology; S Collie, ${ }^{1}$ BSc Hons (Actuarial Science), FASSA; \\ T Chen, ${ }^{1}$ BSc Hons (Actuarial Science), FASSA, CERA; M Cohen, ${ }^{1}$ BBusSc Actuarial Science; \\ J Champion, ${ }^{1}$ MSc Chemical Engineering, PG Diploma Actuarial Science, FASSA; C Feldman, ${ }^{2}$ MB BCh, DSc, PhD, FRCP, FCP (SA); \\ G A Richards, ${ }^{3} \mathrm{MB}$ BCh, PhD, FCP (SA), FRCP, MASSAf
}

${ }_{1}^{1}$ Strategic Risk Management and Data Science Unit, Discovery Health, Johannesburg, South Africa

${ }^{2}$ Department of Internal Medicine, Faculty of Health Sciences, University of the Witwatersrand, Johannesburg, South Africa

${ }^{3}$ Department of Critical Care, Faculty of Health Sciences, University of the Witwatersrand, Johannesburg, South Africa

Corresponding author: S Collie (shirleyc@discovery.co.za)

\begin{abstract}
Background. The phenomenon of silent hypoxaemia has been described in patients with COVID-19 pneumonia, which is characterised by low oxygen saturation levels of $<90 \%$ in those who appear clinically well and do not show signs of significant respiratory distress.

Objectives. To assess the impact on clinical outcomes for high-risk COVID-19 patients using a pulse oximeter to monitor oxygen saturation levels in a home setting.

Methods. We performed a retrospective cohort analysis using data from a large South African insurance administrator. Patients were categorised as high risk, based on age and specific underlying clinical conditions, or from predictive models derived from medical scheme administrative claims data. The impact of pulse oximetry home monitoring on COVID-19 clinical outcomes was investigated by the use of Cox proportional hazard models.

Results. Between 2 March 2020 and 31 October 2020, of 38660 patients analysed, 8115 were in the intervention group. The 60-day mortality rate for the evaluated high-risk population was $1.35 \%$. After adjusting for age and comorbidity differences, the intervention group was found to have an adjusted hazard ratio of $0.52(p<0.0001)$. No statistical significance was found between the intervened and control groups for admission to hospital, admission to intensive care unit (ICU) and use of mechanical ventilation. The intervention group had a lower median C-reactive protein (CRP) level on admission ( $p=0.03$ ). After adjustment for admission CRP levels, elevated CRP was associated with an increased mortality $(p<0.0001)$, while the statistical significance in mortality between the intervention and the control group was lost.

Conclusions. High-risk COVID-19 patients who used a pulse oximeter to monitor oxygen saturation levels had significantly lower mortality rates compared with other high-risk patients. The mortality benefit may be explained by earlier presentation to hospital, as suggested by lower initial CRP levels.
\end{abstract}

S Afr Med J 2021;111(10):950-956. https://doi.org/10.7196/SAMJ.2021.v111i10.15880

The global COVID-19 pandemic was first reported on 31 December 2019 by the World Health Organization (WHO) following a cluster of cases of pneumonia in Wuhan City, Hubei Province, China. ${ }^{[1,2]}$ SARS-CoV-2 was confirmed to be the causative virus of the disease, which was named COVID-19 and declared a global pandemic on 11 March 2020. ${ }^{[1]}$

The phenomenon of silent hypoxaemia has been described in patients with COVID-19 pneumonia, which is characterised by low oxygen saturation levels of $<90 \%$ in patients who appear clinically well and do not show signs of significant respiratory distress. ${ }^{[2,3]}$ Early in the disease process, lung mechanics may be preserved and, although the mechanism is uncertain, it may be related to pulmonary microvascular thrombosis and intrapulmonary shunting analogous to the hepatopulmonary syndrome. ${ }^{[2,4]}$ In this initial phase, respiratory effort is not significantly increased, as the lung compliance remains normal, although the tidal volume may increase markedly in response to the hypoxaemia. Thereafter, extensive alveolar and interstitial inflammation occurs with a marked decrease in pulmonary compliance, which resembles the classic acute respiratory distress syndrome (ARDS). Rapid respiratory decompensation occurs as the compliance decreases, and tachypnoea may be the most important clinical warning sign of impending respiratory failure. ${ }^{[2-6]}$

Monitoring oxygen saturation with pulse oximeters at home can potentially determine the need and appropriate timing for hospital admission more accurately, allowing for early intervention, such as the administration of supplemental oxygen and proning before oxygen saturation drops to $<90 \%$, a level which has been associated with higher in-hospital mortality. ${ }^{[3,7,8]}$ NHS England purchased 200000 of these devices with the intention that they be given to patients at high risk of developing severe COVID-19 for use at home to detect deterioration early and facilitate timeous transfer to hospital, where indicated. ${ }^{\left[{ }^{[]}\right.}$Discovery Health, a large South African (SA) healthcare administrator, similarly provided pulse oximeters to clients with documented comorbidities that had been associated with a high risk of severe COVID-19 illness to evaluate the benefit, if any, of this intervention.

\section{Methods}

This was a retrospective analysis using Discovery Health, an SA managed care organisation's anonymised data, including information 
related to demographics, chronic condition registrations, claims and operational data.

\section{Patient inclusion criteria}

The following inclusion criteria were used:

- a positive SARS-CoV-2 reverse transcriptase polymerase chain reaction (RT-PCR) assay of a specimen collected from the upper or lower respiratory tract before 31 October 2020

- membership of a scheme administered by Discovery Health and high risk of developing severe COVID-19 infection (discussed below)

- acceptance and receipt of a pulse oximeter prior to status, defined as 'concluded' (recovery or death) as per the National Institute for Communicable Diseases (NICD) de-isolation guidelines ${ }^{[10]}$

- concluded COVID-19 status on 17 November 2020.

\section{Patient exclusion criteria}

The following exclusion criteria were used:

- a negative SARS-CoV-2 RT-PCR test result

- a positive SARS-CoV-2 RT-PCR test result, but individuals were not active members of the scheme at the time of data analysis or not classified as high risk.

The following additional exclusion criteria were applied before the data analysis:

- individuals with an unknown RT-PCR collection date

- individuals with missing demographic information

- patients who were admitted or died on the same day as the RT-PCR collection date

- individuals who were contacted and declined a pulse oximeter.

\section{Eligible patients not provided with pulse oximeters}

There were various reasons why many eligible patients were not provided with pulse oximeters: they were either uncontactable, declined to participate, were already being closely monitored by their treating physicians, had already been admitted to hospital, already had access to a personal oximeter and/or were living in an old-age/ care home, where the nursing staff had access to pulse oximeters.

Study patients (intervention arm) were those who fulfilled all the inclusion or exclusion criteria, while the control patients comprised the remainder. Telephonic contact was prioritised for those most at risk of admission within the eligible population, with the remainder receiving email notification of eligibility.

\section{High-risk categorisation}

Patients were categorised as high risk of admission if they had specific underlying clinical conditions, such as diabetes mellitus, cardiovascular conditions (hypertension, ischaemic heart disease), chronic lung disease (asthma, chronic obstructive pulmonary disease), were immunocompromised (HIV, cancer, immunosuppressive treatments, e.g. for autoimmune diseases or after a transplant), had chronic liver disease, were pregnant, or classified as high risk from predictive models based on medical scheme administrative claims data.
A COVID-19 proxy model estimating the risk for admission was used to further categorise those at high risk for severe COVID-19. This model was defined as a composite of the risk for admission and the risk for admission with pneumonia.

The following variables were considered when the models were fitted (Suppl. Figs 1 and 2; http://samj.org.za/public/sup/15880.pdf):

- chronic conditions for which a member was registered

- demographic factors, such as age, sex and type of medical scheme plan purchased

- medications for which a member had claimed in the previous 12 months

- procedures and consultations for which a member had claimed in the previous 12 months

- type of physician practice that member had attended in the previous 12 months

- International Classification of Diseases (ICD) information from claims in the previous 12 months.

Both these models were built in Python using the XGBoost algorithm. Graphs with the variable importance for the different models are provided (Suppl. Figs 1 and 2; http://samj.org.za/public/sup/15880. pdf). The model results were validated by testing the sensitivity and specificity against unseen data, which comprised $30 \%$ of patient records. The precision recall area under the curve (PR-AUC) of the validated test results was $47.8 \%$, and for the training dataset it was $51.9 \%$. Both these models were trained from 1 June 2018 to 31 May 2019. Members who tested positive for COVID-19 and had a $>10 \%$ likelihood of admission risk were flagged as eligible to receive an oximeter. After 17 September 2020, a model was built on COVID-19 admissions to date. This model had improved PR metrics on training data (PR-AUC 58.0\%) and was validated on $30 \%$ of unseen data (PR-AUC 51.5\%). From this model, patients were classified as high risk if they had a minimum of $44 \%$ likelihood of admission risk.

\section{Oximeters}

With regard to those who received oximeters, a wellness specialist followed them up telephonically, explaining how to use the device and interpret the results. Patients were also guided on what to do when abnormal results were observed (Table 1).

Members were provided with a form to record their oxygen saturation (\%) and heart rate (bpm) twice daily. They were also advised to contact their doctor immediately if they had difficulty breathing, regardless of the readings.

\section{Analysis and statistical methods}

Continuous variables were described using means (standard deviation) and, where appropriate, medians (interquartile range). Categorical variables were described as a number (\%). For continuous variables, statistical comparisons were performed with an independent $t$-test for normally distributed data, and a Wilcoxon signed-rank test for non-normally distributed data. The follow-up period for the analysis was 60 days after RT-PCR collection date or until censoring.

Table 1. Pulse oximeter readings and guidance

\begin{tabular}{ll}
\hline Percentage & Guidance \\
\hline$\geq 95 \%$ & Continue to track and record your oxygen saturation levels twice daily during the 2-week period \\
$90-94 \%$ & $\begin{array}{l}\text { If your levels decrease to } 90-94 \% \text { over } 2 \text { readings taken at least an hour apart, you must call your treating general } \\
\text { practitioner for medical assistance }\end{array}$ \\
& If your levels decrease to $<90 \%$ on any reading, or if you have difficulty breathing, get urgent medical care
\end{tabular}


The highest rate of daily deaths and admissions in the high-risk population occurred on the day of PCR collection, and steadily declined each day after that date. The turnaround time of test results among patients and the time taken to dispatch the pulse oximeter were not constant (Suppl. Fig. 3; http://samj.org.za/public/sup/15880. pdf). To avoid bias, mortality outcomes for the intervention group after dispatching the pulse oximeter were compared with those of the control group per day, relative to the PCR collection date, using Cox multivariate analysis.

The following features were included as factors in the Cox multivariate models: age band, number of chronic conditions, hypertension, diabetes mellitus, hypercholesterolaemia, HIV positivity, asthma and an 'intervened indicator' for patients who accepted a pulse oximeter. The top 5 comorbid conditions were selected (based on volume) to support credibility of statistical estimates. Observation times were based on days since the PCR collection date. The hazard ratio of each feature was used to assess the relative impact on mortality risk, as well as the associated $p$-values. Proportionality of hazards for all covariates was checked by observing Schoenfeld residual plots. Statistical analysis was performed using Excel 2016 (Microsoft Inc., USA) and R (version 3.6.3) using the Grammar of Data Manipulation package (dplyr; version 0.8 .3 ) and survival libraries.

\section{Outcome evaluation}

The outcome evaluation was the determination of whether the 60-day mortality rate for the intervention group was better than that of the control group. Furthermore, admission outcomes, including level of ward acuity, length of hospital stay and C-reactive protein (CRP) levels on admission date, were compared between the two groups.

\section{Ethical approval}

Ethical clearance (ref. no. M210149) was obtained from the Human Research Ethics Committee (Medical) of the University of the Witwatersrand, Johannesburg, South Africa.

\section{Results}

Initially, 53038 patients were evaluated for inclusion in the study, 10 862 in the intervention group and 42176 in the control group. After applying the exclusion criteria, 8115 patients remained in the study population, and 30545 in the control population, resulting in 38660 patients included in the final analysis, of whom 586 withdrew from the scheme before the end of the follow-up period (Fig. 1).

Compared with the control group, the intervention group was significantly older, had a higher proportion of males (41\%) and were more likely to have hypertension (34\%), hypercholesterolaemia (21\%), diabetes mellitus (15\%), HIV (8\%) and asthma (12\%) (Table 2). The proportion of patients presenting with the top five registered chronic conditions was also more prevalent in the intervention group (56.3\% v. $42.4 \%$ for control subjects) (Table 2).

The overall 60 -day mortality risk for the population was $1.35 \%$ (1.24 - 1.46\%), representing 544 deaths. Patients $\geq 80$ years old had the highest 60 -day mortality risk (14.59\%; $12.33-16.78 \%)$. Of all chronic conditions evaluated, patients with diabetes had the highest 60 -day mortality risk ( $4.61 \% ; 4.00-5.22 \%)$. The 60 -day mortality rate for the intervened population was $0.68 \%(0.49-0.88 \%)$ compared with $1.44 \%(1.32-1.57 \%)$ for the control group (Table 3$)$.

Adjusting for all covariates in the 30-day Cox mortality model, the following was noted with regard to relative risk (RR) in the evaluated high-risk population (Table 4):

- The study population had a RR of mortality of 0.52 .
- Increased age had a higher risk of mortality, e.g. patients aged 50 59 years had an RR of 1.95 , whereas those aged $\geq 80$ years had an RR of 4.48 .

- Males had an RR of 1.96.

- Diabetic patients had an RR of 2.27.

- Patients with hypercholesterolaemia had an RR of 0.75 .

- Patients with $\geq 3$ chronic conditions had an RR of 1.76 .

- Patients with hypertension, asthma or HIV, and those with one and two chronic conditions, were not associated with significantly increased risk of mortality, given the other variables considered by the model.

A sensitivity analysis was performed to explore whether the RR for the study population would be different in the provinces where COVID19 was most prevalent, i.e. Gauteng and the Western Cape. This represented $79 \%$ of the data. It was found that the study population had an RR of 0.5 . The mortality findings remained consistent with the findings in the entire population, apart from hypercholesterolaemia, which was not associated with an increased risk of mortality.

\section{Risk factors for mortality}

Risk factors were multiplicative relative to the base, where the base consisted of non-intervened lives, female lives and those aged $<40$ years with no chronic conditions (Table 4):

Diabetic males aged 50 - 59 years had a 31.3 times higher mortality risk $(2.27 \times 1.96 \times 7.03)$.

Males aged 70 - 79 years had a 63.5 times higher mortality risk $(1.96 \times 32.38)$, whereas females aged $70-79$ years had a 32.4 times higher mortality risk.

\section{Investigation of factors that may have contributed to a lower risk of death}

The impact of the pulse oximeter intervention on hospital admission, length of stay, need for intensive care unit (ICU) admission or mechanical ventilation and death for the high-risk population was investigated (Table 5). For each of these outcomes, except for length of stay, the impact was assessed by interpreting the hazard ratio from the Cox regression models, including the same demographic risk factors as in the mortality model defined in statistical methods. Mean length of stay was compared between the study and comparator population, and the $p$-value was obtained using the $t$-test.

To assess whether CRP levels were lower for the study population than the control group, the one-sided Wilcoxon rank-sum test was performed.

\section{Impact of the intervention on outcomes}

The impact of the pulse oximeter intervention on clinical outcomes is shown in Table 5 .

Three hundred and twenty-one patients in the study population were admitted to hospital. The impact of the intervention resulted in a non-significant 3\% higher likelihood of admission compared with the control group $(p=0.59)$. The mean length of stay for the intervention group was 8.03 days compared with 7.45 days for the control group $(p<0.0001)$.

Overall, 49 patients in the study group died, but if the mortality was equivalent to that of the controls, 95 (95\% confidence interval (CI) 70.5 - 128.1; $p<0.0001$ ) deaths would have been expected, i.e. 46 fewer deaths in the pulse oximeter group than in the control group (95\% CI 21.5 - 79.1; $p<0.0001$ ) (Table 3).

There was no significant difference between admission to ICU, requirement for mechanical ventilation or extracorporeal membrane 


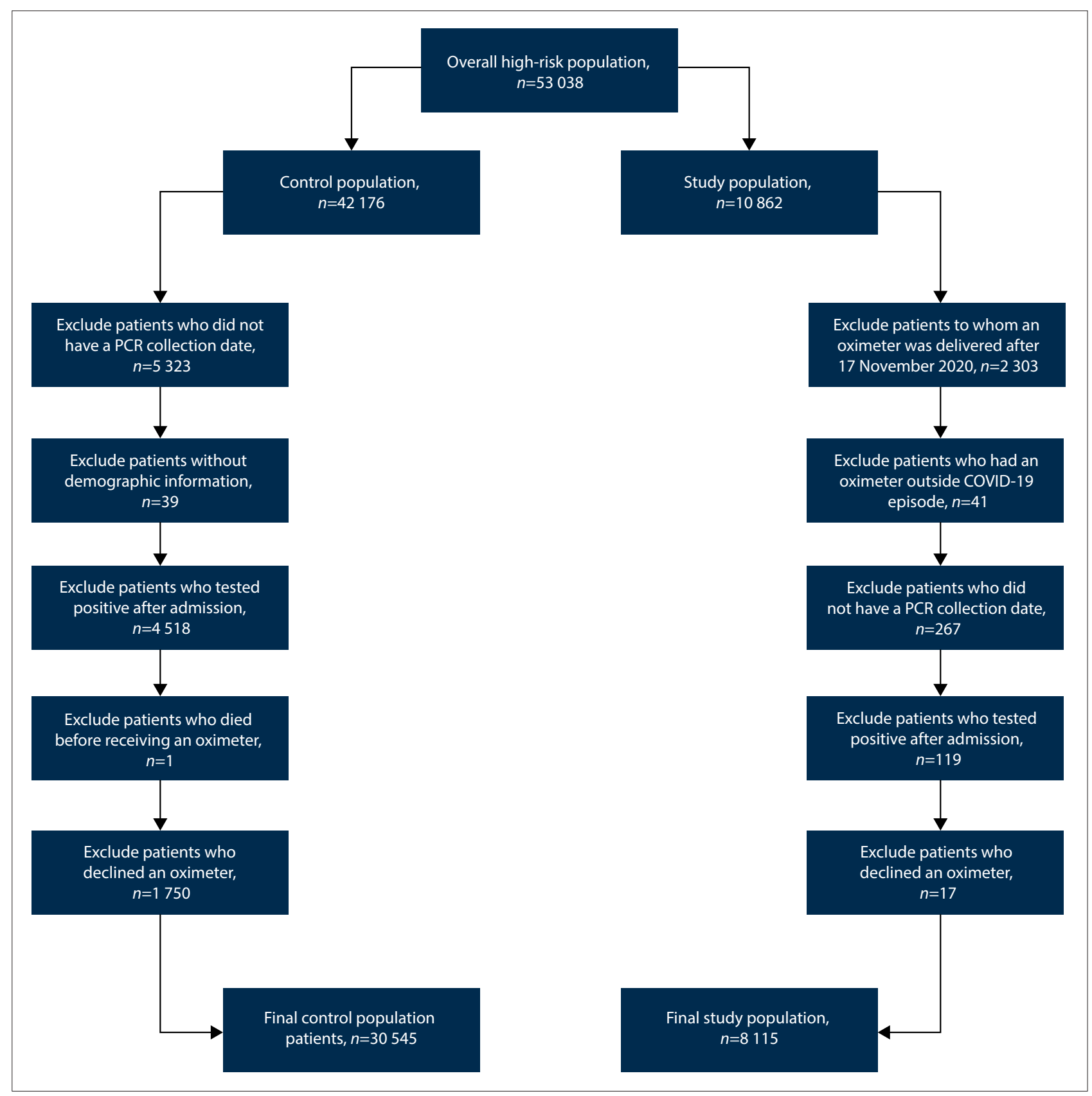

Fig. 1. Study profile. $(P C R=$ polymerase chain reaction. $)$

\begin{tabular}{llll} 
Table 2. Baseline characteristics & & \\
\hline & Study population $(\mathbf{N = 8 ~ 1 1 5 ; ~ 2 1 \% )}$ & Control population $(\mathbf{N = 3 0 ~ 5 4 5 ; ~ 7 9 \% )}$ & $p$-value \\
\hline $\begin{array}{l}\text { Age (years), mean (SD) } \\
\text { Sex, } n \text { (\%) }\end{array}$ & $46.4(15.2)$ & $43.7(16.6)$ & $<0.0001$ \\
$\quad$ Male & $3341(41)$ & $11704(38)$ & $<0.0001$ \\
$\quad$ Female & $4774(59)$ & $18841(62)$ & $<0.0001$ \\
Most common conditions, $n$ (\%) & & & $<0.0001$ \\
$\quad$ Hypertension & $2753(34)$ & $7882(26)$ & $<0.0001$ \\
Hypercholesterolaemia & $16789(21)$ & $4250(14)$ & 0.03 \\
$\quad$ Diabetes mellitus & $1177(15)$ & $3199(10)$ & $<0.0001$ \\
HIV & $615(8)$ & $2504(8)$ & $2070(7)$ \\
$\quad$ Asthma & $990(12)$ & &
\end{tabular}




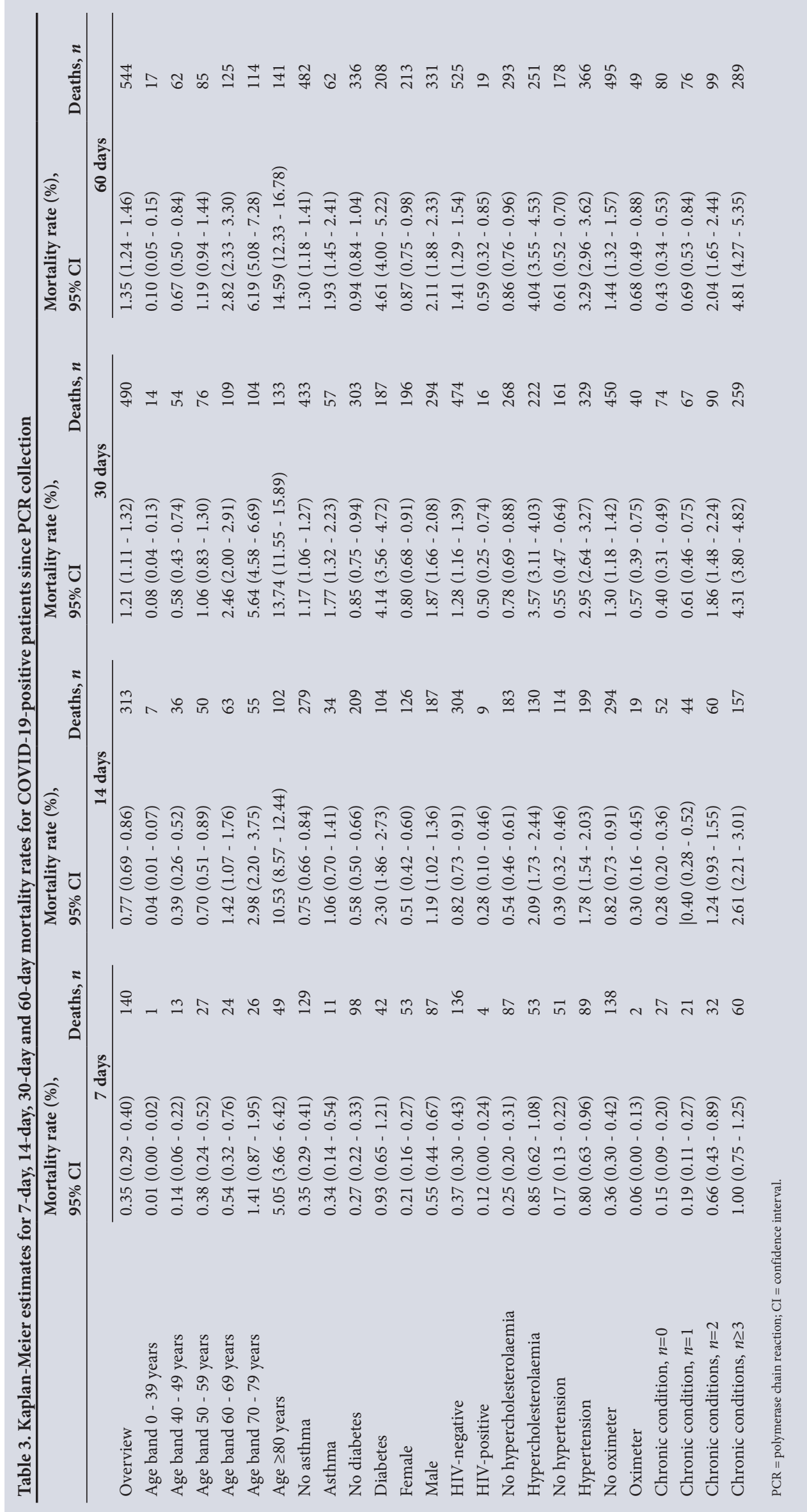

oxygenation (ECMO) between the intervention and control groups.

CRP pathology results on admission to hospital were available for $238(74 \%)$ of the 321 patients and 2115 (58\%) of the 3642 admitted in the study and control populations, respectively (Table 6). The median CRP result, $56 \mathrm{mg} / \mathrm{L}$ in the study group, was lower than that in the control group ( $70 \mathrm{mg} / \mathrm{L} ; p=0.03$ ).

Using this result as a covariate in the Cox mortality model (Table 4), the study population had no statistically significant difference in mortality $(p=0.18)$, whereas the CRP test result did have a significant impact on mortality $(p<0.0001)$ (Suppl. Table 1; http:// samj.org.za/public/sup/15880. pdf). Each additional unit increase in the CRP had a $0.53 \%$ increase in mortality risk $(p<0.0001)$. Therefore, a patient admitted with the population median CRP result of 68.3 , would have a $43.6 \%$ (1.01 $\times 68.3)$ higher mortality risk.

\section{Discussion}

The aim of providing patients at high risk of severe COVID19 infection with pulse oximeters was to improve clinical outcomes by earlier identification of silent hypoxaemia or a deterioration in oxygen saturation, thus decreasing the acuity of admissions, the requirement for intensive care and mechanical ventilation, and the number of deaths. The data adjusted for risk showed a statistically significant improvement in 60-day mortality (hazard ratio $0.52 ; p<0.0001$ ) for the intervention group, despite this group consisting of older patients, with a higher prevalence of the top five comorbid conditions, who should have been at higher risk for worse outcomes.

While there was a trend toward a higher likelihood for admission for the intervention group, this was not statistically significant (hazard ratio $1.03 ; p=0.59$ ). For those in the intervention group who were admitted, a longer mean length of stay was observed (mean difference 0.58 days; $p<0.0001$ ).

In a single-centre retrospective cohort study that included patients 
Table 4. Cox regression results for deaths

\begin{tabular}{llllll}
\hline & Estimate & Hazard ratio & Lower 95\% CI & Upper 95\% CI & $p$-value \\
\hline Intervened & -0.66 & 0.52 & 0.38 & 0.70 & $<0.0001$ \\
Age band 40 - 49 years & 1.57 & 4.79 & 2.78 & 8.24 & $<0.0001$ \\
Age band 50 - 59 years & 1.95 & 7.03 & 4.12 & 12.00 & $<0.0001$ \\
Age band 60 - 69 years & 2.78 & 16.06 & 9.50 & 27.15 & $<0.0001$ \\
Age band 70 - 79 years & 3.48 & 32.38 & 18.96 & 55.29 & $<0.0001$ \\
Age band $\geq 80$ years & 4.48 & 87.85 & 51.64 & 149.47 & $<0.0001$ \\
Male & 0.67 & 1.96 & 1.64 & 2.34 & $<0.0001$ \\
Hypertension & 0.24 & 1.27 & 0.99 & 1.62 & 0.06 \\
Hypercholesterolaemia & -0.28 & 0.75 & 0.60 & 0.95 & 0.02 \\
Diabetes & 0.82 & 2.27 & 1.85 & 2.79 & $<0.0001$ \\
HIV & 0.12 & 1.13 & 0.70 & 1.84 & 0.62 \\
Asthma & 0.12 & 1.13 & 0.85 & 1.49 & 0.402 \\
Chronic condition, $n=1$ & -0.09 & 0.92 & 0.65 & 1.30 & 0.63 \\
Chronic conditions, $n=2$ & 0.35 & 1.42 & 0.98 & 2.06 & 0.06 \\
Chronic conditions, $n \geq 3$ & 0.57 & 1.76 & 1.16 & 2.67 & 0.01
\end{tabular}

Table 5. Summary of impact of pulse oximeter intervention on clinical outcomes

\begin{tabular}{|c|c|c|c|c|c|}
\hline & Rate per patient & $\begin{array}{l}\text { HR for impact of the intervention } \\
\text { on the study population }\end{array}$ & Lower 95\% CI & Upper 95\% CI & $p$-value \\
\hline Admission ${ }^{*}$ & $10.098 \%$ & 1.03 & 0.92 & 1.17 & $0.59^{\dagger}$ \\
\hline Length of stay* & 7.50 days & 1.078 & 1.05 & 1.093 & $<0.0001^{*}$ \\
\hline $\mathrm{ICU}^{s}$ & $1.650 \%$ & 1.02 & 0.80 & 1.31 & $0.86^{\dagger}$ \\
\hline Mechanical ventilations & $0.904 \%$ & 0.97 & 0.69 & 1.36 & $0.86^{\dagger}$ \\
\hline ECMO & $1.419 \%$ & 0.52 & 0.38 & 0.70 & $<0.0001^{\dagger}$ \\
\hline Mortality & $1.49 \%$ & 0.52 & 0.38 & 0.70 & $<0.0001^{\dagger}$ \\
\hline \multicolumn{6}{|c|}{$\begin{array}{l}\text { HR = hazard ratio; } \mathrm{CI}=\text { confidence interval; } \mathrm{ICU}=\text { intensive care unit; } \mathrm{ECMO}=\text { extracorporeal membrane oxygenation. } \\
{ }^{*} \text { Excludes } 330 \text { patients in the study group who were admitted before receiving a pulse oximeter, resulting in } 7783 \text { remaining patients in the group. } \\
\text { 'p-value obtained from intervention hazard ratio from Cox multivariable models for each of the analysed outcomes. } \\
\text { " } t \text {-test based on mean difference in average length of stay between the study and control populations. } \\
\text { 'Excludes } 30 \text { patients in the study group who were admitted to an ICU before receiving a pulse oximeter, resulting in } 8083 \text { remaining patients in the study group. } \\
\text { 'Excludes } 23 \text { patients in the study group who had mechanical ventilation or ECMO before receiving a pulse oximeter, resulting in } 8090 \text { remaining patients in the study group. }\end{array}$} \\
\hline
\end{tabular}

Table 6. Total number of patients admitted, with a CRP test on admission date

\begin{tabular}{|c|c|c|c|c|}
\hline & Study population & Control population & Total, $n$ & $p$-value \\
\hline Admissions, $n(\%)$ & $321(8)$ & $3642(92)$ & 3963 & \\
\hline CRP test results available on day of admission, $n(\%)$ & $238(10)$ & $2115(90)$ & 2353 & \\
\hline Median CRP test result (interquartile range), mg/L & $56(21.2-111.1)$ & $70(20.5-141.7)$ & $68.3(20.5-138.1)$ & $0.03^{*}$ \\
\hline
\end{tabular}

with moderate to critical COVID-19 pneumonia hospitalised in Wuhan, $25.7 \%$ died during hospitalisation after a median 14-day follow-up. In this study, higher oxygen saturation levels after oxygen supplementation were associated with reduced mortality, independently of age and sex. ${ }^{[7]}$ Access to a pulse oximeter for monitoring oxygen saturation allows patients to detect deterioration timeously and seek healthcare.

Patients who accepted a pulse oximeter may have been more conscientious and aware of symptoms and may potentially have been more likely to seek care timeously, regardless of having a pulse oximeter. The effect, however, is expected to be small, as most members who were contacted accepted the device (83\% telephonic acceptance rate).

In a systematic review, Figliozzi et al.$^{[11]}$ identified clinical history and laboratory profile as vital to identification of patients with a higher risk of in-hospital mortality. Various severity scores have also been evaluated in COVID-19 for the prediction of in-hospital clinical outcomes, including death. ${ }^{[12]}$ Using these scores at the time of admission could help to identify patients at higher risk of worse clinical outcomes at the time of admission.

Elevated inflammatory markers are associated with severe COVID19 disease. In a systematic review and meta-analysis by Yamada et al. ${ }^{[13]}$ and a study by Luo et al., ${ }^{[14]}$ elevated CRP levels on admission were associated with severe illness and poor clinical outcomes. In our study, of the patients with a CRP test result available on the day of admission, the median CRP in the oximeter group was significantly lower (56 $\mathrm{mg} / \mathrm{L})$ than that of controls $(70 \mathrm{mg} / \mathrm{L} ; p=0.03)$, and could therefore explain the lower mortality in the group that received a pulse oximeter. This shows that these patients had less severe disease at the time of admission than the control group (median difference $14 \mathrm{mg} / \mathrm{L} ; p=0.03$ ). 


\section{Study limitations}

This was a retrospective analysis of medical aid records and was therefore not structured as a clinical trial at the outset. There may have been patients among the control group who had already used a pulse oximeter, resulting in them being incorrectly allocated to one of the study groups. However, as the use of a pulse oximeter was associated with a favourable mortality outcome, patients with pulse oximeters incorrectly allocated to the control group would have lowered the overall mortality risk for that group, whereas, despite this possibility, the mortality was significantly lower in the intervention group.

Corticosteroids, and in particular dexamethasone, are the only therapies conclusively shown to lower mortality in hospitalised patients with COVID-19 who receive invasive mechanical ventilation or oxygen alone. These therapies are, therefore, not indicated for use in the outpatient setting, unless there is evidence of hypoxaemia. ${ }^{[15,16]}$ The timing of the initiation of corticosteroids and other treatments in hospitalised patients could not be determined from the available data and therefore the differences between the groups could not be established.

It is possible that those who received a pulse oximeter could have had higher admission oxygen saturation levels than the control group and received oxygen sooner, which could explain the mortality benefit. These data were, however, not available for evaluation.

Another factor that might have been relevant was body mass index (BMI), which is an independent risk factor for COVID-19related deaths, with a $48 \%$ higher risk of mortality ${ }^{[17-19]}$ BMI data were, however, not available for comparison between the two groups. Access to hospital health records would have provided more detail regarding poor prognosticatory factors that may have contributed to the mortality difference observed between the two groups, including BMI data.

Future prospective studies should be undertaken to further evaluate the benefits of pulse oximeter intervention in improving mortality outcomes. Patients included in this study are members of a private medical insurance in SA and have access to better healthcare resources than the majority of patients, who mainly rely on resource-constrained government facilities for care. Because of these disparities in access to healthcare, the study results may not be completely generalisable to the public health setting in SA.

\section{Conclusions}

Home monitoring using a pulse oximeter in patients at high risk of developing severe COVID-19 infection resulted in a $48 \%$ lower likelihood of death relative to the control group $(p<0.0001)$. Higher CRP test results were associated with an elevated mortality risk of $0.53 \%(p<0.0001)$ per unit increase. Of the individuals with a CRP test result available on the day of admission, the median CRP in the oximeter group $(56 \mathrm{mg} / \mathrm{L})$ was significantly lower than that of the controls $(70 \mathrm{mg} / \mathrm{L} ; p=0.03)$. The mortality difference of the study population could only be explained by these relatively lower CRP levels, which were a surrogate for disease severity compared with the control population (median difference $14 \mathrm{mg} / \mathrm{L} ; p=0.03$ ).

\section{Declaration. None.}

Acknowledgements. Discovery Health administered scheme boards for approving the pulse oximeter benefit for eligible members of their schemes. We thank Discovery Health's Dr Ryan Noach for his leadership and support; the Data Science Unit for data support; and the Discovery
Operations teams and Discovery Clinical Services for the operational management of the pulse oximeter benefit.

Author contributions. TC, MC, JC and SC identified patients and collected relevant data. MC, TC and SC ensured that the data were quality checked and complete. SC and NN were responsible for the implementation of analysis. TC, JC, NN, SC, CF and GAR interpreted the results. MC, TC and SC performed the statistical analysis. SC and NN created the initial drafts of the manuscript. SC, NN, CF and GAR were responsible for essential revisions of the manuscript and for incorporation of important intellectual content. All authors reviewed the manuscript and gave final approval for submission.

\section{Funding. None.}

\section{Conflicts of interest. None.}

Data sharing. De-identified patient data will be made available for any purpose, beginning 6 months and ending a minimum of 36 months after publication of this article, to researchers who provide a methodologically sound proposal, whose proposed use of the data has been approved by an independent review committee identified for this purpose. Proposals may be submitted beginning 6 months and up to 36 months after publication of this article. Proposals should be directed to shirleyc@discovery.co.za; to gain access, data requestors will need to sign a data access agreement.

1. World Health Organization. https://www.who.int/emergencies/diseases/novel-coronavirus-2019/ events-as-they-happen (accessed 20 December 2020).

2. Dhont S, Derom E, van Braeckel E, et al. The pathophysiology of 'happy' hypoxemia in COVID-19. Respir Res 2020;21:198. https://doi.org/10.1186/s12931-020-01462-5

3. Caputo ND, Strayer RJ, Levitan R. Early self-proning in awake, non-intubated patients in the emergency department: A single ED's experience during the COVID-19 pandemic. Acad Emerg Med 2020;27(5):375-378. https://doi.org/10.1111/acem.13994

4. Reynolds AS, Lee AG, Renz J, et al. Pulmonary vascular dilatation detected by automated transcranial Doppler in COVID-19 pneumonia. Am J Respir Crit Care Med 2020;202(7):10371039. https://doi.org/10.1164/rccm.202006-2219LE

5. McGonagle D, O’Donnell JS, Sharif K, Emery P, Bridgewood C. Immune mechanisms of pulmonary intravascular coagulopathy in COVID-19 pneumonia. Lancet Rheumatol 2020;2(7):e437-e445. https://doi.org/10.1016/S2665-9913(20)30121-1

6. Gattinoni L, Chiumello D, Caironi P, et al. COVID-19 pneumonia: Different respiratory treatments for different phenotypes? Intens Care Med 2020;46(6):1099-1102. https://doi.org/10.1007/s00134020-06033-2

7. Xie J, Covassin N, Fan Z, et al. Association between hypoxemia and mortality in patients with COVID-19. Mayo Clin Proc 2020;95(6):1138-1147. https://doi.org/10.1016/j.mayocp.2020.04.006 8. Sun Q, Qiu H, Huang M, et al. Lower mortality of COVID-19 by early recognition and intervention: Experience from Jiangsu Province. Ann Intens Care 2020;10(1):33. https://doi.org/10.1186/s13613020-00650-2

9. Torjesen I. Covid-19: Patients to use pulse oximetry at home to spot deterioration. BM] 2021;372:n677. https://doi.org/10.1136/bmj.n677

10. National Institute for Communicable Diseases. https://www.nicd.ac.za/wp-content uploads/2020/08/Clinical-management-of-suspected-or-confirmed-COVID-19-V5-24August-2020.pdf (accessed 21 December 2020).

11. Figliozzi S, Masci PG, Ahmadi N, et al. Predictors of adverse prognosis in COVID-19: A systematic review and meta-analysis. Eur J Clin Invest 2020;50(10):e13362. https://doi.org/10.1111/eci. 13362 12. Fan G, Tu C, Zhou F, et al. Comparison of severity scores for COVID-19 patients with pneumonia: $A$ retrospective study. Eur Respir J 2020;56(3):2002113. https://doi.org/10.1183/13993003.02113-2020

13. Yamada T, Wakabayashi M, Yamaji T, et al. Value of leukocytosis and elevated C-reactive protein in predicting severe coronavirus 2019 (COVID-19): A systematic review and meta-analysis. Clin Chim Acta 2020;509:235-243. https://doi.org/10.1016/i.cca.2020.06.008

14. Luo X, Zhou W, Yan X, et al. Prognostic value of C-reactive protein in patients with Coronavirus 2019. Clin Infect Dis 2020;71(16):2174-2179.
.

15. The RECOVERY Collaborative Group. Dexamethasone in hospitalized patients with Covid-19. N Engl J Med 2021;384(8):693-704. https://www.nejm.org/doi/10.1056/NEJMoa2021436

16. Cohen P, Blau J. Coronavirus disease 2019 (COVID-19): Outpatient evaluation and management in adults. UpToDate Oct 2020. https://www.uptodate.com/contents/covid-19-outpatient-evaluationand-management-of-acute-illness-in-adults (accessed 14 August 2021).

17. Huang Y, Lu Y, Huang YM, et al. Obesity in patients with COVID-19: A systematic review and meta-analysis. Metabolism 2020;113:154378. https://doi.org/10.1016/j.metabol.2020.154378

18. Popkin BM, Du S, Green WD, Beck MA, et al. Individuals with obesity and COVID-19: A global Popkin BM, Du S, Green WD, Beck MA, et al. Individuals with obesity and COVID-19: A global
perspective on the epidemiology and biological relationships. Obes Rev 2020;21(11):e13128. https://doi.org/10.1111/obr.13128

19. Kompaniyets L, Goodman AB, Belay B, et al. Body mass index and risk for COVID-19-related hospitalization, intensive care unit admission, invasive mechanical ventilation, and death - United States, March - December 2020. MMWR Morb Mortal Wkly Rep 2021;70(10):355-361. https://doi. org/10.15585/mmwr.mm7010e4

Accepted 6 August 2021. 